\title{
Beeinflusst sportliche Aktivität die Revisionsrate nach Knietotalendoprothese? Analyse eines 12-Jahres-Follow-up
}

\section{Does Sports Activity Influence Total Knee Arthroplasty Durability? Analysis with a Follow-Up of 12 Years}

Autoren

Christina Valle, Michael Sperr, Christina Lemhöfer, Karla Elida Bartel, Marcus Schmitt-Sody

Institut

Orthopädie, Medical Park Chiemsee, Bernau, Deutschland

Schlüsselwörter

Knie, Totalendoprothese, Sport, Revision

Key words

knee joint, sports, total knee arthroplasty, revision

Bibliografie

DOI http://dx.doi.org/10.1055/s-0043-103007 |

Sportverl Sportschad 2017; 31: 111-115

(c) Georg Thieme Verlag KG Stuttgart · New York

ISSN 0932-0555

Korrespondenzadresse

Dr. med. Christina Valle

Orthopädie, Medical Park Chiemsee

Birkenallee 41

83233 Bernau am Chiemsee

Deutschland

c.valle@medicalpark.de

\section{ZUSAMMENFASSUNC}

Hintergrund Der Anspruch von Patienten an Knietotalprothesen (KTEP) wächst zunehmend. Es stellt sich immer wieder die Frage, ob sportlich aktive Patienten nach Implantation einer KTEP ein erhöhtes Revisionsrisiko aufweisen im Vergleich zu Patienten ohne sportliche Aktivitäten.

Patienten/Material und Methoden In einer retrospektiven Studie wurden 130 Patienten mit einem Altersdurchschnitt von 69,2 Jahren zu Ihrer sportlichen Aktivität 22 Monate vor und nach KTEP Primärimplantation befragt. Die Patienten wurden aufgrund der Ergebnisse der Befragung in 2 Gruppen ( $A=$ Sport, $B=$ No-Sport) eingeteilt. 7 Jahre postoperativ wurden dieselben Patienten erneut zu Ihrem Sportverhalten und 12 Jahre postoperativ zusätzlich zu stattgehabten Revisionsoperationen befragt.

Ergebnisse Von den 130 im Jahre 2005, ca. 2 Jahre nach KTEP-Implantation, befragten Patienten gaben 88 (67,7\%) an, regelmäßig Sport auszuüben (Gruppe A) während 42 Patienten (32,3\%) keinerlei Sport betrieben (Gruppe B). 2010 antworteten 87 der 130 Patienten auf die Befragung.
60 Patienten der Gruppe A waren weiterhin regelmäßig sportlich aktiv. 27 Patienten der Gruppe B gaben an, weiterhin keinerlei Sport zu betreiben. 2015 konnten von den 87 Patienten die 2010 befragt wurden immerhin noch 67 Patienten kontaktiert werden - davon 46 Patienten der Gruppe A und 21 Patienten der Gruppe B. In Gruppe A kam es 12 Jahre postoperativ bei 7 von 46 Patienten (15,2\%) zu Revisionsoperationen. In Gruppe B gaben 5 von 21 (23,8\%) Patienten Revisionsoperationen im Sinne von Wechseloperationen in den letzten Jahren an.

Schlussfolgerung Es konnte gezeigt werden, dass 12 Jahre nach Primärimplantation einer KTEP die Revisionsrate in der Gruppe der sportlich aktiven Patienten verglichen mit den Inaktiven deutlich reduziert war. Daher lässt sich schlussfolgern, dass moderate sportliche Aktivität nach künstlichem Kniegelenkersatz keinen negativen Einfluss auf die Standzeit einer Knietotalendoprothese hat, sondern sogar möglicherweise zu verbesserter Osteointegration und damit zu weniger osteolytischen Reaktionen mit daraus resultierender aseptischer Prothesenlockerung führen könnte.

\section{ABSTRACT}

Background Total knee replacement is the only surgical option to treat painful degenerative osteoarthritis, restore knee function, improve quality of life, and enable patients to return to sports activity. With an aging population expecting an active life after retirement, patients' expectations of improvement after surgery are increasing as well. In view of the growing demand for higher performance, exercise and athletic activity, clinicians have been increasingly forced to question how much athletic activity a patient may resume after total knee replacement, and what types of athletic activity seem to be acceptable. However, there is currently no consensus on the appropriate intensity of physical activity after total knee arthroplasty or how activity levels affect the rate of revision surgery.

Patients, material and methods In a retrospective study with a follow-up period of 12 years, 130 patients were asked about their sport activities 22 months before and after total knee replacement. Based on their answers, the patients were divided into one sports group (group A) and one group with- 
out any sports activity (group B). Seven years after surgery the same patients were asked about their sports activity again, and 12 years postoperatively they were additionally asked about whether or not they underwent revision surgery.

Results Twenty-two months after total knee replacement 88 (67.7\%) of the 130 patients practiced sports for a minimum of 3 times a week (group A), whereas 42 (32.3\%) patients reported that they practiced no sports (group B). In 2010 - 7 years postoperatively - 60 patients of group $A$ and 27 patients of group B took part in the survey; their levels of sports activity had not changed over the previous 7 years. In 2015, 46 patients out of group A and 21 patients out of group B were questioned. We saw a revision rate of $15.2 \%$ (7 out of 46 patients) in the group with continuous sports activity, whereas we found an increased revision rate of $23.8 \%$ (5 out of 21 patients) in the group without any sports activity 12 years after total knee arthroplasty.

Conclusion Our study showed that sports activity after total knee replacement led to a reduced revision rate in the sports group compared with the non-sports group. Therefore, more liberal recommendations should be given to patients regarding athletic activity after total knee arthroplasty. We conclude that moderate sports activities have no negative influence on total knee arthroplasty and may even lead to improved osteointegration with a decrease in osteolytic changes and less prosthesis loosening.

\section{Einleitung}

Die Implantation einer Knietotalendoprothese (Knie-TEP) gehört zu den häufigsten Operationsverfahren weltweit und steht mit 127077 Eingriffen in Deutschland 2013 an 15. Stelle der durchgeführten stationären Operationen [1]. Die häufigste Indikation stellt die primäre Gonarthrose dar. Aufgrund der längeren Lebenserwartung und Lebensqualität der Patienten steigt auch der Anspruch an die Prothesenhaltbarkeit und -funktion. Obwohl die Standzeit der Knie-TEP mit ca. 95\% nach 10 Jahren sehr gut ist und damit mit der der Hüft-TEP vergleichbar ist [2], ist die Patientenzufriedenheit nach Knie-TEP deutlich schlechter [3]. Als Behandlungsziel geben viele Patienten nicht nur Schmerzfreiheit, sondern auch die volle Funktionsfähigkeit inklusive Rückerlangen der Sportfähigkeit bzw. den Wunsch nach sportlicher Aktivität, uneingeschränkter Mobilität und Unabhängigkeit auch in höherem Lebensalter an. In unserer modernen Gesellschaft ist Sport ein wichtiger Bestandteil des Lebens, der sich positiv auswirkt auf die Gesundheit, die Lebensqualität, soziale Kontakte sowie das Selbstwertgefühl. Gerade bei älteren Patienten gewinnt der Sport zunehmend im Sinne der sozialen Integration an Bedeutung. Verschiedene Arbeitsgruppen konnten zeigen, dass die sportliche Aktivität nach Implantation einer KTEP zunimmt [4]. Dies ist in erster Linie der postoperativen Schmerzfreiheit und der damit verbundenen gesteigerten Patientenzufriedenheit geschuldet. Durch die zunehmende Aktivität scheint sich auch das funktionelle Ergebnis zu verbessern, was durch EMG-Untersuchungen von Erler et al. gezeigt werden konnte [5]. Die positiven Auswirkungen sportlicher Aktivität auf die Lebensqualität sind unbestritten, aber auch die modernsten Implantate halten dauerhaft nicht allen Belastungen stand. Gerade in den letzten Jahren wird deshalb zunehmend kontrovers diskutiert, inwiefern sportliche Betätigung und vor allem deren Art und Intensität Einfluss auf die Lebensdauer einer Knie-TEP hat. Beim Gehen in der Ebene können Kniegelenkskräfte vom 3-4fachen, beim abwärts gehen sogar vom 8fachen des Körpergewichtes auftreten. Beim Joggen steigen die Belastungen sogar bis auf das 12 fache des Körpergewichtes an. So berichten die meisten Arbeitsgruppen über einen erhöhten Abrieb und damit verbunden eine gesteigerte aseptische Lockerungsrate von Kunstgelenken durch vermehrten Aktivitätslevel mit einhergehenden gesteigerten Bewegungszyklen [6]. In vitro zeigte sich bereits bei alltäglichen Belastungen wie Gehen oder Treppensteigen eine erhöhte Abriebrate der Inlays von Knie-TEP's [7].

Auf der anderen Seite wird postuliert, dass regelmäßige sportliche Aktivität mit relativ geringer bis mittlerer Gelenkbelastung zumindest mittelfristig mit einem Follow-up von 4 Jahren keinen negativen Einfluss auf die Standzeit von künstlichen Hüftgelenkersatz zu haben scheint [8]. Empfohlen werden daher vor allem sogenannte Low-impact-Sportarten wie Fahrradfahren, Wandern oder Schwimmen, um eine möglichst lange Prothesenstandzeit zu erreichen [9]. Neben dem Aktivitätslevel und patientenspezifischen Einflüssen spielt sicherlich die Implantatwahl, die exakte Positionierung sowie die Operationstechnik eine entscheidende Rolle. Zusätzlich gilt es zu bedenken, dass allein wegen der in den letzten Jahren konstant hohen Implantationsrate und der zeitgleich zunehmenden Lebenserwartung das Revisionsrisiko nach Implantation einer Knie-TEP ansteigt.

Es stellt sich also die Frage, ob eine vermehrte mechanische Belastung einer Prothese durch sportliche Aktivität zu einer höheren Revisionsrate führt. Wie sich die sportliche Aktivität nach Jahren im Langzeitverlauf in Bezug auf Abrieb, Lockerung oder mechanische Komplikation der Knie-TEP auswirkt, ist nach wie vor nicht vollständig geklärt.

Daher war es Ziel dieser Arbeit mit einem Langzeit-Follow-up von 12 Jahren zu untersuchen, welchen Einfluss sportliche Aktivität auf die Standzeit und damit Revisionsrate der Knie-TEP hat.

\section{Patienten/Material und Methoden}

In unserer retrospektiven Studie wurden im Jahr 2005130 Patienten (68 männlich und 62 weiblich) mit einem Durchschnittsalter von 69,2 Jahren mittels standardisiertem Fragebogen zu Ihrer sportlichen Aktivität vor und nach Knie-TEP-Implantation befragt. Zu diesem Zeitpunkt lag die Implantation einer zementierten, ungekoppelten, bikondylären Knie-TEP ohne Patellarückflächenersatz durchschnittlich 22 Monate zurück. Patienten mit bereits postoperativ aufgetretenen Komplikationen wie persistierende Schmerzen von größer 2 auf der NRS, Folgeoperationen oder Funktionseinschränkungen wie Extensionsdefizit oder Flexionsfä- 
higkeit von weniger als $100 \mathrm{Grad}$ am betroffenen Kniegelenk wurden von der Studie ausgeschlossen.

Die Patienten wurden entsprechend den Ergebnissen im Fragebogen in eine Sportgruppe (= Gruppe A) und in eine No-Sportgruppe (= Gruppe B) unterteilt. Gruppe $A$ gab an, regelmäßig sportlich aktiv zu sein. Gruppe B hingegen übte 22 Monate nach Endoprothesenimplantation keinerlei Sport aus.

2010, durchschnittlich 7 Jahre nach Knie-TEP Implantation, wurden die gleichen 130 Patienten erneut telefonisch befragt, ob sie weiterhin regelmäßig sportlich aktiv waren, welche Sportarten sie ausführten und ob es zu Komplikationen im Sinne von Revisionsoperationen gekommen ist. Nur bei gleichbleibender sportlichen Aktivität 2005 und 2010 wurden die Patienten in den Gruppen A (Sport) bzw. B (No-Sport) weitergeführt.

Sowohl 2005 wie auch 2010 wurde nach Befragung der Patienten der Hospital for Special Surgery Knee Score (HSS Score) entsprechend der Gruppenzugehörigkeit bestimmt.

2015, durchschnittlich 12 Jahre nach Knie-TEP Implantation, wurden die 2005 und 2010 befragten Patienten erneut telefonisch interviewt. Diesmal wurden etwaig stattgehabte Revisionsoperationen in Abhängigkeit der Gruppenzugehörigkeit 2005 und 2010 berücksichtigt. Nur Patienten, die sowohl 2005 wie auch 2010 der gleichen Gruppe zugeordnet werden konnten, kamen 2015 in die Auswertung.

\section{Ergebnisse}

Bei den 2005 befragten Patienten waren die fünf häufigsten präoperativ durchgeführten Sportarten Radfahren, Schwimmen, Wandern im Flachen, Bergwandern und Ski Alpin. Circa 22 Monate nach KTEP-Implantation gaben 88 Patienten (66,7\%) an, regelmäßig Sport zu betreiben (Gruppe A-Sport), während 42 Patienten (32,3\%) keinerlei Sport betrieben (Gruppe B-No-Sport). Es handelte sich dabei nahezu ausschließlich um Sportarten mit geringer und mittlerer Gelenkbelastung ( $\triangleright$ Table 1 ). Mehrfachnennungen waren möglich. 2010 antworteten 87 der 130 im Jahre 2005 befragten Patienten. Dies entspricht einer Rücklaufquote von 66,9\%. 60 Patienten der Gruppe A trieben weiterhin regelmäßig Sport. 27 Patienten der Gruppe B gaben an, weiterhin keinerlei Sport zu betreiben. Auch 2010, 7 Jahre nach Knie-TEP Implantation wurden mit nahezu gleicher Häufigkeit die entsprechenden Sportarten durchgeführt ( $\bullet$ Table 1 ).

Der HSS-Score der Patienten stieg sowohl in Gruppe A-Sport wie auch in Gruppe B-No-Sport im Jahre 2010 leicht an. Die sportlich aktiven Patienten zeigten sowohl 2005 wie auch 2010 einen besseren HSS Score verglichen mit den inaktiven Patienten ( Table 2).

2015 konnten von den 87 Patienten, die 2010 befragt wurden, immerhin noch 67 Patienten kontaktiert werden. Davon konnten 46 Patienten der Gruppe A und 21 Patienten der Gruppe B zugeordnet werden. Dies entspricht einer Rücklaufquote von $77 \%$ ( $\triangleright$ Table 3 ).

In Gruppe A kam es 12 Jahre postoperativ bei 7 von 46 Patienten (15,2\%) zu Revisionsoperationen. In der Gruppe B gaben 5 von 21 (23,8\%) Patienten Revisionsoperationen im Sinne von Wechseloperationen in den letzten Jahren an.
- Tab. 1 Prozentuale Darstellung der ausgeübten Sportarten mit Knie-TEP 2 Jahre (2005) und 7 Jahre (2010) postoperativ.

\begin{tabular}{|l|l|l|}
\hline Sportart & $\begin{array}{l}\text { Häufigkeit } \% \\
\mathbf{2 0 0 5}\end{array}$ & $\begin{array}{l}\text { Häufigkeit } \% \\
\mathbf{2 0 1 0}\end{array}$ \\
\hline Wandern & $48,3 \%$ & $42,5 \%$ \\
\hline Radsport & $43,4 \%$ & $37,5 \%$ \\
\hline Schwimmen & $42,9 \%$ & $35,0 \%$ \\
\hline Golfen & $18,3 \%$ & $15,0 \%$ \\
\hline Nordic walking & $16,9 \%$ & $12,5 \%$ \\
\hline Skilanglauf & $13,7 \%$ & $15,0 \%$ \\
\hline Ski alpin & $12,3 \%$ & $10,0 \%$ \\
\hline
\end{tabular}

- Tab. 2 HSS-Score 2005 und 2010: Gruppe A (Sport), Gruppe B (No-Sport) MW \pm SEM.

\begin{tabular}{|l|l|l|}
\hline HSS-Score & Gruppe A-Sport & Gruppe B-No-Sport \\
\hline 2005 & $83,44 \pm 13,04$ & $72,6 \pm 16,18$ \\
\hline 2010 & $85,38 \pm 9$ & $74,22 \pm 14,95$ \\
\hline
\end{tabular}

- Tab. 3 Gruppenzugehörigkeit 2005 - 2015 ( = Anzahl der Patienten).

\begin{tabular}{|l|l|l|}
\hline & Gruppe A-Sport & Gruppe B-No-Sport \\
\hline 2005 & 88 & 42 \\
\hline 2010 & 60 & 27 \\
\hline 2015 & 46 & 21 \\
\hline
\end{tabular}

\section{Diskussion}

Die Anzahl Sport treibender Personen in unserer Gesellschaft steigt aufgrund des sozialen Wandels mit geändertem Freizeitverhalten und geändertem Körperbewusstsein kontinuierlich an [10]. Aufgrund des demografischen Wandels wird Sport auch zunehmend in höherem Alter ausgeübt, weshalb die Frage nach sportlicher Aktivität nach künstlichem Gelenkersatz auch immer mehr in den Vordergrund rückt.

Ob und mit welcher Intensität Sport nach künstlichem Gelenkersatz ausgeübt werden kann oder sollte, ist nach wie vor nicht abschließend beantwortet und wird in der aktuellen Literatur kontrovers diskutiert $[11,12]$. Es gibt Arbeiten, die eine reduzierte sportliche Aktivität nach künstlichem Gelenkersatz [11], andere wiederum ein gesteigertes Aktivitätslevel postoperativ zeigen [13]. Sicherlich ist dies auch abhängig vom präoperativen Aktivitätslevel [14]. Die derzeitigen Patientenempfehlungen orientieren sich lediglich an Konsensusaussagen oder Befragungen der Operateure [15]. 
Bei sportlich aktiven Patienten wirken deutlich höhere Krafteinwirkungen und Scherkräfte auf die Implantate. Des Weiteren zeigen sich ein verstärkter oberflächlicher Gelenkabrieb sowie ein erhöhtes Risiko traumatischer Verletzungen des Kniegelenkes, die zu gehäuftem Auftreten von Osteolysen und Prothesenversagen führen können, verglichen mit nicht aktiven Patienten [1619].

So konnte Kilgus et al. eine deutlich gesteigerte Revisionsrate nach Hüft-TEP Implantation von 28 \% bei sportlich aktiven Patienten im Vergleich zu $6 \%$ bei nicht aktiven Patienten nachweisen, wobei die Revisionsrate erst nach 10 Jahren signifikant anstieg [20]. Somit könnte Sport einen negativen Effekt auf die Haltbarkeit der Prothesenkomponenten haben und damit zu vermehrten Abrieb führen, der als ursächlich für die aseptische Lockerung angesehen wird.

Im Gegensatz dazu zeigten andere Arbeitsgruppen einen positiven Effekt sportlicher Aktivität auf das Knochenwachstum und die Osteointegrität von Prothesenkomponenten [21]. Es besteht Einigung darüber, dass verbesserte Knochenqualität die Prothesenfixation verbessert und damit die Lockerungsrate senkt $[22,23]$.

Dubs et al. untersuchten 110 Patienten [24] 5,8 Jahre nach Hüft-TEP-Implantation. Die häufigsten postoperativ durchgeführten Sportarten waren vergleichbar mit denen aus unserer Studie: Wandern (41\%), Schwimmen (35\%), Jogging (17\%) und Fahrradfahren (10\%). In dieser Untersuchung fand sich eine signifikant niedrigere Revisionsrate knapp 6 Jahre postoperativ von 1,6\% in der Sportgruppe verglichen mit der No-Sportgruppe von 14,3\%. Inwiefern diese Ergebnisse auch im Langzeitverlauf zutreffen und auf künstliche Kniegelenke übertragbar sind, ist bisher nicht geklärt.

In der Literatur finden sich sehr wenige prospektive Studien mit sehr kurzem Follow-up. Auch der Follow-up der publizierten retrospektiven Studien zu Sport nach künstlicher Knie-TEP reicht zur Beantwortung der Frage nach Haltbarkeit bzw. Revisionsrate nicht aus.

Unsere Ergebnisse 12 Jahre nach Knie-TEP Implantation zeigen eine Revisionsrate von $23,8 \%$ in der nicht aktiven Gruppe und von $15,2 \%$ in der sportlich aktiven Gruppe. Ob der niedrigere HSSScore in der nicht aktiven Gruppe ursächlich ist für die sportliche Inaktivität oder die fehlende sportliche Aktivität zu einem niedrigeren HSS-Score führte, bleibt letztendlich unbeantwortet. Inaktivität kann zu Osteoporose führen [25]. Inwiefern im Umkehrschluss sportliche Aktivität die Knochendichte und damit die Verankerung der Prothese verbessert und somit Frühlockerungen entgegenwirken könnte, lässt sich anhand der Ergebnisse der vorliegenden Arbeiten nur vermuten, allerdings nicht abschließend beantworten.

Unser gewähltes, retrospektives Studiendesign ist einem prospektiven Untersuchungsansatz sicherlich unterlegen und führt daher auch zu einem insgesamt relativ hohen Drop-out, der sich allerdings aufgrund des gewählten Alterskollektivs mit einem Follow-up von 12 Jahren wiederum relativiert. Die Umstände, die zum Drop-out oder zur Revision führten, sind meist nicht klar erfasst aufgrund z. T. fehlender Erreichbarkeit der Patienten oder/ und nicht vollständig ausgefüllter Fragebogen. Ein weiterer Schwachpunkt ist sicherlich die Inhomogenität der Probanden.
So wurden nicht alle Patienten vom gleichen Operateur und auch nicht mit dem gleichen Implantat versorgt. Die von den Patienten gewählten Sportarten prä- und postoperativ entsprechen zum Teil der alpennahen Wohngegend und können ggf. nicht auf andere Patienten übertragen werden. Nach unserer Auffassung spiegelt das gewählte Studiendesign ebenso wie das Patientenkollektiv, trotz der aufgeführten Schwächen, dennoch die Realität des klinischen Alltags wieder und bietet daher möglicherweise eine hilfreiche Antwort auf die Fragen vieler Patienten, ob sportliche Aktivität auch über 10 Jahre hinaus der Haltbarkeit von künstlichen Kniegelenken schadet oder nicht.

\section{Schlussfolgerung}

Es konnte gezeigt werden, dass 12 Jahre nach Primärimplantation einer Knie-TEP die Revisionsrate in der Gruppe der sportlich aktiven Patienten verglichen mit den inaktiven deutlich reduziert war. Daher lässt sich schlussfolgern, dass moderate sportliche Aktivität nach künstlichem Kniegelenkersatz keinen negativen Einfluss auf die Standzeit der Knieprothese hat und im Gegenteil sogar möglicherweise zu verbesserter Osteointegration und damit zu weniger osteolytischen Reaktionen mit daraus resultierender aseptischer Prothesenlockerung führen könnte. Sportartspezifische Empfehlungen nach künstlichem Kniegelenkersatz sind sicherlich von verschiedenen Faktoren abhängig und müssen unter anderem das Alter des Patienten, den Prothesentyp, Komorbiditäten, sportliche Vorerfahrungen und den Rehabilitationsverlauf sowie das funktionelle Ergebnis berücksichtigen.

\section{Interessenkonflikt}

Es besteht seitens der Autoren kein Interessenkonflikt.

\section{Literatur}

[1] AQUA Institut für angewandte Qualitätsförderung und Forschung im Gesundheitswesen GmbH. Bundesauswertung zum Erfassungsjahr, 17/5 - Knie-Totalendoprothesen-Erstimplantation. 2013

[2] Lützner ], Hübel U, Krischner $S$ et al. Langzeitergebnisse in der Knieendoprothetik. Der Chirurg 2011; 82: 618-624

[3] Bourne RB, Chesworth B, David A et al. Comparing patient outcomes after THA and TKA: is there a difference? Clin Orthop Relat Res 2010; 468: $542-546$

[4] Bock P, Schatz K, Wurnig C et al. Körperliche Aktivitäten nach Knietotalendoprothesenimplantation. Z Orthop Unfall 2003; 141 (3): 272-276

[5] Erler K, Neumann U, Anders C et al. Nachuntersuchungsergebnisse mittels EMG-Mapping - 5Jahre nach Knieendoprothesenimplantation. Z Orthop Unfall 2003; 141 (1): $48-53$

[6] Harris WH. Wear and periprosthetic osteolysis: the problem. Clin Orthop Relat Res 2001; 393: 66

[7] Schwiesau J. „Knee wear simulation under conditions of highly demanding daily activities-influence on an unicompartmental fixed bearing knee design“. Med Eng Phys 2013; 35 (8): 1204-1211

[8] Mont MA, Marker DR, Seyler TM et al. High-impact sports after total knee arthroplasty. J Arthroplasty 2008; 23: 80 
[9] Wimmer MD. „Sport nach Hüft- und Knieendoprothetik“. Schweizerische Zeitschrift für Sportmedizin und Sporttraumatologie 2012; 60 (2): $80-82$

[10] Healy WL, Sharma S, Schwartz B et al. Athletic activity after total joint arthroplasty. J Bone Joint Surg Am 2008; 90 (10): 2245 - 2252

[11] Huch K, Müller KA, Stürmer T et al. Sports activities 5 years after total knee or hip arthroplasty: the Ulm study. Ann Rheum Dis 2005; 64 (12): $1715-1712$

[12] Wylde V, Blom A, Dieppe P et al. Return to sport after joint replacement. J Bone Joint Surg Br 2008; 90 (7): 920 - 923

[13] Jackson JD, Smith J, Shah JP et al. Golf after total knee arthroplasty: do patients return to walking the cours? Am J Sports Med 2009; 37 (11): $2201-2204$

[14] Mont MA, Rajadhyaksha AD, Marxen JL et al. Tennis after total knee arthroplasty. Am J Sports Med 2002; 30 (2): 163-166

[15] Bauman S, Williams D, Petruccelli D et al. Physical activity after total joint replacement: a cross-sectional survey. Clin J Sport med 2007; 17 (2): $104-108$

[16] Schmalzried TP, Shepherd EF, Dorey FJ et al. Wear is a function of use, not time. Clin Orthop Relat Res 2000; 381: 36-46

[17] Lavernia C], Sierra JR, Hungerford DS et al. Activity level and war in total knee arthroplasty: a study of autopsy retrieved specimens. J Arthroplasty $2001 ; 16: 446-453$
[18] Kuster MS, Stachowiak GW. Factors affecting polyethylene wear in total knee arthroplasty. Orthopedics 2002; 25 (2): s235-s242

[19] D 'Lima DD, Patil S, Steklov N et al. Tibial forces measured in vivo after total knee arthroplasty. J Arthroplasty 2006; 21: 255-262

[20] Kilgus DJ, Dorey FJ, Finerman GAM et al. Patient activity, sports participation, and impact loading on durability of cemented total hip replacements. Clin Orthop 1991; 269: 25-31

[21] Widhalm R, Höfer G, Krugluger ] et al. Ist die Gefahr der Sportverletzung oder die Gefahr der Inaktivitätsosteoporose beim Hüftträger größer? Folgerungen auf die Dauerhaftigkeit von Prothesenverangerungen. Z Orthop 1990; 128: 139-143

[22] Kuster MS. Exercise recommendations after total joint replacement: a review af the current literature and proposal of scientifically based guidelines. Sports Med 2002; 32 (7): 433 - 445

[23] Swanson EA, Schmalzried TP, Dorey F]. Activity revommendations after total hop and knee arthroplasty: a survey of the American Association for Hip and Knee Surgeons. J Arthroplasty 2009; 24 (6): 120-126

[24] Dubs L, Gschwend N, Munzinger U. Sport after total hip arthroplasty. Arch Orthop Trauma Surg 1983; 101: 161 - 169

[25] Widhalm R, Höfer G, Krugluger ] et al. Ist die Gefahr der Sportverletzung oder die Gefahr der Inaktivitätsosteoporose beim Hüftträger größer? Folgerungen auf die Dauerhaftigkeit von Prothesenverangerungen. Z Orthop 1990; 128: 139-143 